Spargelgläser nach dem Sterilisieren. Sobald dic Gläser einigermaßen abgekühlt sind, bringe man sie sofort in einen Kübel mit kaltem Wassen, das man mehrmals ergänzt, oder stellt am besten der Kübel unter eine Leitung und läbt mohrere Stunden frisches Wasser daruber rieseln. Dieses Auskühlen ist auch beim Nachsterilisieren zu wiederholen und bei Erbsen und Bohnen ebenfalls empfehlenswert." (.,Die Frischhaltuag" 1917, 7 , s. 17.)

Bei eine* derantigen geringen thermischen Widerstandsfähigkeit der jetzigen Handelsgläsen ist es kein Wunder, dab die Konserrenindustrie, die je nach Art der Konserve bei $1-3$ Atmosphären $\left(120-113^{\circ} \mathrm{C}\right)$ im Autoklavea sterilisient wnd dann die eben berausgenommenen Büchsen womöglich noch mit kaltem Wasser abbraust, bis jetzt nicht daxan denken konnte, Glasbüchsen im groben Mabstabe als Ersatz für Biechbüchsen zu verwenden. Aber nicht allein die geringe thermische, sondem auch die geringe chemische Wrderstandsfähigkeit, die sich, je höher die Sterilisationstemperatur liegt, um so störender bemerkbar macht, hindert die allgemeine Benutzung dev Glasbüchse in der Industrie. Auf diesen Mangel macht schon Ott mit folgenden Worten aufinerksam:

„Die wichtigste Eigenschaft für Konserven gläser . Ist ihre Widerstandsfähigkeit gegen Wasser, Säuren und Salzlösungen. Absolut widerstandsfahig ist kein Glas gegen diese Agentien, aber durch richtige Zusammensetzung des Glases, vamentlich bezüglich des Kalkgehaltes, läßt sich die Widerstandsfähigkeit leicht beträchtlich steigern. Wie stark unter Unständen die Einwirkung ron Konserven aut das Glas werden kam, beweist ein Fall, den Professor Weber analysiert hat. Er fand bei einem solchen sterilisierten Konservenglas die Oberfläche mit Gruppen von Kristallen belegt, die er als Zeolitbildung (Hydrosilikat) erkannte. Unter allen Umständen empfiehlt es sich, die Konservengläser, namentlich die für Gemüse und feine Früchte bestimmten, entweder in mit Salzsäure angesäuertem Wasser tüchtig auszukochen, oder noch besser, wenn irgend möglich, sie durch strömenden Wasserdampf zu reinigen, wodurch das Glas eine in Wasser fast unlösliche kieselsäurereiche Oberflächenschicht erhält" (Dr. I. Ott, .Die Fabrikation der Gemüsekonserven", Wien 1909, S. 57.)

Welche Zerstoruagen schon durch mehrmaliges Sterilisieren im Hausgebrauch bei $100-105^{\circ}$ an Konservengläsern bewirkt werden, zeigen nebenstehende Abbildungen. Man sieht hier einen dem Glasfachmann bei minderwertigen Gläsern unter der Bezeichnung Quellung bekannten Vorgang. Durch Einwirkung des flüssigen Wassers und Wasserdampfes tritt eine Zersetzung des Glases ein, die unter Bildung von Rissen und Absplittern mehr oder minder großer Teile vor sich geht. Versuche mit den Konservengläsern aus Jenaev Glas exgaben, dab trotz oft wiederholtem Sterili- sieren in tutoklaren bei etwa $120^{\circ}$ keine ler artigen Vtründerungen festzustellen waren.

Aus vorstehenden Ausfuhrungen dürfte hervorgehen, daB die jetzt im Handel befindlichen Konservengläser, selbst bei vorsichtiger Behandlung, kaum den Anforderungen des Hausgebrauchs bei dem jetzt üblichen Konservierungsrerfalren genügen. Bei jeder Art der Konservierung, die im Intexesse eines schnelleren Arbeitens oder einer besseren Sterilisierung nur wenig höhere Anforderungen stellt, wärden sie sofort vollständig versagen. Es würde z. B. ein groBer Vorteil in bezug auf Ersparnis an Brennmaterial, bessere Sterilisation und schnelleres Arbeiten sein, wenn man Fleisch und Gemüse, statt im Wasserbad, im Backofen sterilisiêren könnte. Dies scheitert aber an der Mangelhaftigkeit der jetzigen Gläser. Auch die Konservenindustrie würde bessere Konserven, z. B. Früchte, Prinzeßbohnen usw. - fur die große Menge wird stets der Blechbichse der Vorzug gegeben werden in Gläsern konservieren können, wenn ein Glas vorhanden wäre, das den zu stellenden Anforderungen genügen würde. Das Bedürfnis nach besseren Konservengläsern ist also wohl zweifellos vorhanden.

\section{Europas meteorologische Hochstationen vor dem Kriege.}

Von Prof. Dr. F. Klengel, Plauen i. V.

Wit Ausnahme ron Griechenland und der Türkei sowie von einigen kleinen Ländern besaßren alle Staaten Europas vor Ausbruch des Weltkrieges einen öffentlichen meteorologischen Beobachtungsdienst. Die Zahl aller diesem Dienste angehörenden Stationen wird man auf 21500 schätzen können. Enter diesen befanden, sich etwa 2100 Stationen 1. und 2. Ordnung, die mit allen nötigen Werkzeugen zum Ablesen oder zur selbsttätigen Aufzeichnung von Witterungserscheinungen ausgestattet waren. Weitere 600 besaben nur einen Teil dieser Instrumente (Stationen 3. Ordnung), die übrigen waren in der Hauptsache nur mit dem Messen des atmosphärischen Niederschlags beschäftigt. Eine beträchtliche Zahl dieser Beobachtungspunkte gehört den Gebirgen an, denn die Erforschung der höheren Luftschichten ist schon seit dem internationalen Meteorologenkongre $B$ in Rom, seit 1879, dex wichtigste Bestandteil aller auf dje $\mathbf{E r}-$ kundung der atmosphärischen Vorgänge gerichteten Untersucbungen geworden.

Teilt man die Stationen nach Höhenstufen ron $500 \mathrm{zu} 500 \mathrm{~m}$ ein, so ergibt sich, das mehr als 660 Stationen über $1000 \mathrm{~m}$ hoch, rund 150 von ihnen über $1500 \mathrm{~m}, 44$ über $2000 \mathrm{~m}, 8$ über $2500 \mathrm{~m}$ und 1 Station über $3000 \mathrm{~m}$ hoch liegen. $\mathrm{F}_{\mathrm{S}}$ ist also ein stattliches Netz ron Höhenstationen, das vor Kriegsausbruch Europa bedeckte, und es dürte wenige Gebirge gegeben 
haben, in densin nicht Barometer und Thermometer oder wenigstens Regenmesser in größeren Iföhen aufoestellt worden waren. Allerdings sind in vorstehenden Zahlen auch eine größere Anzahl von ,Sommerstationen" inbegriffen, die ibre Tätigkeit nur wähend der warmen Monate ausübten. Dies gilt vor allem von verschiedenen Hochstationen der Alpen und der norwegischen Gebirge, die in den nur monateweise geöffneten Schutzhïtten und Unterkunftshäusern untergebracht sind. Den gröBten Anteil an diesem Netz haben selbstverständlich die Alperländer: Osterreich, die Schweiz, Frankreich, Bayern und Ttalien. Von den 2800 Stationen des österreichir schen Beobachtungssystems befinden sich über 260 in der Höhenlage von mehr als $1000 \mathrm{~m}$ und noch 21 in einer solchen ron mebr als $2000 \mathrm{~m}$. Die höchste ron allen und zugleich die höchste dauernd tätige in ganz Europa ist das Observatorium anf jem Sonnblick, $3106 \mathrm{~m}$, in den hohen Tanern. In der Schweiz gehönen vollends über $25 \%$ aller Stationen der Höhenlage ron melar als $1000 \mathrm{~m}$ an. Von diesen 105 Stationen rageu noch 8 in die Zone von $2000 \mathrm{~m}$ hinein, abel nur eine, das Observatorium auf dem Gipfel des Säntis, epreicht die Höhe ron $2500 \mathrm{~m}$. Nicht inbegriffen sind in diesen Ziffern die sogenannten ,Totalisatoren $^{*}$, d. h. SammelgefäBe für den Niederschlag mit Jahresfüllung. Seit dem Sommer 1913 werden nämlich an einer Reihe houhgelegener Punkte der Schweiz, zum Teil weit oberhalb der Firngrenze, große Zinkgefäbe aufgestellt, die zur andauernden Sammlung der Niederschläge hestimmt sind. Uber diese Einrichtung hat der Sohweizer Meteorologe Maurer ${ }^{1}$ mehrfach interessante Mitteilungen gemacht, aus denen wir die folgenden Einzelheiten entnchmeu: Der Gedanke. durch groBe SammelgefäBe in der Schweiz den Niederschlag für längere Zeiträume aulzuspeichern und zu bestimmen, ist nicht neu. Die ersten Versuche in dieser Richtung wurden bereits gegen Ende des vorigen Jahrhunderts durch. die Gletscherkommission der Schweizer naturforschenden Gesellschaft auf Betreiben ihres Präsidenten Eduard IIagenbach im Gebiete des Rhonegletschers, $2600 \mathrm{~m}$, angestellt. Mau verwandte hierzu grope labische, wasserdicht schlieBende Kisten von 1 am Öffung. Die Ergebnisse waren aber offenbar nicht befriedigend und rechtfertigten die hohen Kosten und groBen Mühen des Aufbaus dieser Forschungsmittel in keiner Weise. Ahnliche Versuche wurden etwas später von amerikanischen Meteorologen in den Gebirgen Nordamerikas unternommen. Sie benutzten umfangreiche, mit besonderen Windschutzrorrichtungen ausgestattete Schneekisten, deren. Tanlenlange $1 \frac{1}{2} m$ betrug. In diese hinein wurden erst die eigentlichen SammelgefäBe gestellt, die man mit iI und Salz beschickte, um die Verdunstung zu verhindern und das Anftauen des

1) Met. Zeitschr. 1915, S. 16, und .Das Wetter" conderheft zu ABmanns 70. Geburtstag, 1915, S. 39.
Schnees zu bewirken oder zu erleichtern. Auch mit diesen Apparaten verfolgte man den Zweok, an weit entlegenen und im Winter schwer zugängTicken Punkten des Hochobirges Regen und Schnee lange Zeit aufzuspeichern, um zu gelegener Zeit die Sammelgefäße wieder aufzusuchen und ihren Inhalt zu messen. In der Schweiz wurden die nach dem gleichen Ziele gerichteten Versuche seit 1910 durch den savoyischen Forstinspektor Mougin wieder aufgenommen. Es gelang ihm, Niederschlagssammler zu konstruieren, die es gestatten, den Schnee und Regen monatelang, ja sogar 1 Jahr lang za bewahren. so dal nur in der günstigen Jahreszeit eine Messung notwendig wird. Ein Totalisator, System Mougin. ist ein Zinkblechgefäß von $50 \mathrm{~cm}$ Durchmesser, 95 cm Hohe und einer oberen freien Offnung von $16 \mathrm{~cm}$ Durchmesser (Hellmannring). Die Auffangfläche beträgt also $200 \mathrm{~cm}^{2}$, bei der 1 I Schmelzuasser einer Niederschlagshöhe von $50 \mathrm{~mm}$ entspricht. Insgesamt würde der Inhalt cines solchen Sammlers eine Niederschlagshohe von $4000 \mathrm{~mm}$ darstellen. Das Gefäß wird nun. um das andauernde Auftauen des Schnees zu bewirken, vorher mit einer Chlorcalciumlösung beselickt, und zwar nimmt man gewöhnlich $5 \mathrm{~kg}$ $\mathrm{CaOl}_{2}$ auf 5-6 1 Wasser. Durch diese Lösung wird der Schneeniederschlag, auch bei sehr tiefen AuBentemperaturen von $-30^{\circ}$ in ässeriger Form gehalten; eine Decke von Vaselinöl (1 1). das ebenfalls vorher dem Gefäb zugefügt wird, hat die Aufgabe, die Verdunstung der Schmelzflüssigkeit zu verhindern. Um endlich die stöjeaden Einwirkungen des Windes aufzuheben. the eine Verminderung der in Schneeform gefallenen Niederschläge una $20 \%$ hervortufen können, wurden die Niederschlagssammler mit einem geeigneten Windschutzring (Nipherseher Form) versehen. Dieser Windschutz hat 100 bis $120 \mathrm{~cm} \cdot$ Durchmesser und besitzt die Gestalt eines abgestumpften Kegels. Genaue Vergleiche eines dergestalt ausgestatteten Totalisator mit einem täglioh abgelesenen Stationsregenmesser haben in einem Fall einen tberschuß der Jahressumme voll ca. $100 \mathrm{~mm}$ bei dem ersteren gegenüber dem letzteren ergeben, weil bei der einmaligen Ablesung der Mouginapparate die unvermeidlichen kleinen Fehler der täglichen Ablesung bei den gewöhnlichen Apparaten in Wegfall kommen. Die mit dem Niederschlagssammler festgestellten Werte können daher als recht zuver]äsigig angesehen werden. Was die $A b-$ lesung am Totalisator anbelangt, so würde die Gewichtsbestimmung des Gesamtinhalts nach $\mathrm{Ab}$ lauf eines Jahres wohl "die sicherste Form der Messung bedenten. Fs mübte vom Gesamtgewicht natürlich das Gewicht der $\mathrm{CaCl}_{\text {a-Lösung und des }}$ Vaselinöls abgezogen werden. Da aber das Mitführen einer Wage in die Hochregionen mit groBen Schwierigkeiten verbunden ist, so wird die Gewichtsemithung durch eine Bestimmung des Folumens ersetzt, die wesentlich einfacher ist. 
Allerdings entsteht dabei ein Fehler, weil ursprünglich eine gesättigte $\mathrm{CaCl}_{2}$-Lösung im Gefäf vorhanden war, die allmäblich durch den Niederschlag immer mehr verdünnt wird. Die hiermit verbundene Kontraktion des Volumens stellt den Messungsfehler dar, der jedoch nur stwa 0,5 bis $1 \%$ Verlust gegenüber dem durch Wägung gewonnenen Ergebnis bedeutet und daher nicht in Betracht kommt. Die ersten Ergebnisse dieses Verfalurens, im Jahre 1914. waren so günstig, daß die Zahl der Messungsstellen in den nächsten Jahren noch wesentlich erhoht wurde. So wurden 19162 Totalisatoren in Höhen über $3000 \mathrm{~m}$ aufgestellt (an den Diablerets $3248 \mathrm{~m}$ und am Col d'Orny $3150 \mathrm{~m}$ ), 7 weitere in einer Lage von 2500 bis $3000 \mathrm{~m}$ und 4 in einer Höhe von $2000-2500 \mathrm{~m}$. Wie Maurer mitteilt, sind die oberhalb $3000 \mathrm{~m}$ gemessenen Mengen auffallend grob, so daB die Anschauungen vom Vorhandensein einer Maximalzone des Niederschlags bedeutend unterhalb der Firngrenze, wenigstens in diesem Teile der Alpen, eine Abänderung erfahren werden.

Auch Frankreich besitzt die stattliche Reihe von 110 Höhenstationen, die teils den Alpen, teils den Pyrenäen und einigen französischen Mittelgebirgen angehören. Frankreichs höchste, dauernd betriebene Station ist das berühmte Observatorium auf dem Gipfel des $P$ ic du Midi in den Pyrenäen, $2859 \mathrm{~m}$. Fast ebenso hoch liegt das Observatorium auf dem Mt. Mounier, $2740 \mathrm{~m}$, in den Seealpen, nordwestlich von. Nizza, während unter $2000 \mathrm{~m}$. Höhe die bekannten, zum Teil schon lange tätigen Observatorien auf dem Mont Ventoux, $1900 \mathrm{~m}$, dem Aigoual, $1567 \mathrm{~m}$, und dem Puy de Dôme sich befinden. Das Vallot-Observatorium, $450 \mathrm{~m}$ unterhalb des Gipfels des Mont Blane (auf den Bosses du Dromadaire, $4358^{\circ} \mathrm{m}$ ), wird dagegen nur vorübergehend, im Sommer für besondere wissenschaftliche Untersuchungen, auch außerhalb des Rahmens der meteorologischen Forschung benutzt.

Italien verfügte nach den letzten Angaben, die allerdings etwas weiter zurückliegen, über 20 Hohenstationed, ron denen noch 5 uber die Zone von $2000 \mathrm{~m}$ hinausragen. Höchste Station ist das Observatorium auf dem Atna, $2942 \mathrm{~m}$, in Sizilien, während das Osservatorio Regina Margherita auf dem Monte Rosa, $4560 \mathrm{~m}$, nur zu vorübergehendem Aufenthalt im Sommer geoffnet ist. $\mathrm{Zu}$ den $28^{1}$ ) bayerischen Höheastationen gehört die zweithöchste Europas, das Observatorium auf dem Zugspitzgipfel, $2962 \mathrm{~m}$, das nunmehr bald auf eine 20-jährige ununterbro. chene Tätigkeit zurückblicken kann. Die Ergebnisse dieser höchsten deutschen Wetterwarte sind zu Beginn des Krieges von dem Münchener MeteoroJogen $\mathrm{Dr}^{\mathrm{r}}$. Huber in einer überaus wertvollen Arbeit veröffentlicht worden ${ }^{2}$ ).

1) Die meisten davon sind allerdings nur Niederschlagsstationen.

2) A, Huber: Das Klima der Zugspitze, MUnchen
AuBerhalb der Alpen besitzt Deutschland in den Mittelgebirgen noch weitere 10 Höhenstationen, von denen die Station a der Schneekoppe, $1602 \mathrm{~m}$, am höchsten aufragt. Während des Krieges entstand unter bedentendem Kostenaufwand auf dem höchsten Berg Sachsens, dem Fichtelberg im Erzgebirge, $1215 \mathrm{~m}$, ein neves Observatorium, las 1916 seine Tätigkeit begann bzw. wieder aufnahm. Eine größere Zahl hochgelegener Stationen (54) findet man auch noch in Spanien, doch ist unter ihnen keine Gipfelstation. Die höchste. Prados de Cuence, ist $1660 \mathrm{~m}$ hoch. Portugal gab fruher eine Station in der Sierra de Estrella, $1217 \mathrm{~m}$, als höchstgelegene an. Doch fehlen seit 1895 weitere Mitteilungen darüber. Von den Balkanlabdern haben sowohl Serbien wie auch Bulgarien und Rumänien einige über $1000 \mathrm{~m}$ hoch gelegene Stationen, aber auch die höchste, Palais Sitniakowo in Bulgarien, bleibt noch unter 1800 Meter. Weit wichtiger sind die 19 Höhenstationen Bosniens und der Herzegowina, unter denen das Observatorium auf der Bjelašnica fast bis zu 2100 m aufragt. Ungarn hat in den Karpathen eine Anzahl Bergstationen errichtet. Seit 1905 befindet sich auch auf einem der höchsten Punkte der Beskiden, auf der Babjagora, in dem $1616 \mathrm{~m}$ hoch gelegenen Unterkunftshaus der Sektion Bielitz des Beskidenvereins ein meteorologischer Posten.

Von den nordischen Ländern besitzt nur Norwegen in der Bergregion eine Anzahl Beobachtungspunkte. Vollkommen ausgerüstet ist jedoch nur Finse, $1224 \mathrm{~m}$, während die höher gelegenen, wie Juvashytten, $1840 \mathrm{~m}$, ausschlieblich Niederschlagsmessungen während des Sommers ausfüren. Schwedens höchste Station liegt dagegen nur $593 \mathrm{~m}$ hoch. Es ist die bekannte Wetterdienststation Storlien an der Bahn StockholmTrondhjem.

GroBbritanniens höchste Wetterwarte auf dem Ben Nevis, $1343 \mathrm{~m}$, in Schottland ist leider 1904 nach 20-jähriger Tätigkeit wieder geschlossen worden. Thre Ergebnisse sind nicht nur fast alljährlich in der Met. Zeitschrift veröffentlicht, sondern auch in zusammenfassender Darstellung von $W m$. T. Kilgour in dem interessanten, mit zahlreichen Abbildungen ausgestattetèn Buche Twenty years on Ben Nevis erörtert worden.

\section{Mineralogisch - petrographische Mitteilungen.}

Die schwierige Frage, ob in den Zeollthen das Wasser chemisch gebunden oder nur in physikaliseher Anlagerung enthalten ist, beschaftigt $G$. Stollossa (Neues Jahrb. f. Miner. usw. Beil., Bd. 42, 1918, S. 1 bis 64). Man hatte bis jetzt nach den Untersuchungen von Friedel fast allgemein angenommen, da $B$ die $Z$ Zeolithe das Wasser in sich wie in einem Schwamm aufunehmen vermögen, weil man bei den Entwässe-

1914. Sonderabdiruck aus „Beob. der met. Stat. im Kgrr. Bayern's Bd. 35, 1913. 